\title{
Understanding the Interplay between Task Scheduling, Memory and Performance
}

\author{
Germán Ceballos, Erik Hagersten, David Black-Schaffer \\ Uppsala University, Sweden \\ firstname.lastname@it.uu.se
}

\begin{abstract}
New programming models have been introduced to aid the programmer dealing with the complexity of large-scale systems, simplifying the coding process and making applications more scalable. Task-based programming is one example that became popular recently. At the same time, understanding performance of multicore systems is key for getting faster execution times and optimize workloads, but it is becoming harder due to the increased complexity of hardware and the runtime systems.

To address this, we present TaskInsight, a technique to characterize the memory behavior of different task schedulers through the analysis of data reuse. TaskInsight provides high-level information that can be correlated with tasks' performance variation over time to understand data reuse through the caches due to scheduling choices. This allows to identify which scheduling decisions affected performance, when were they taken, and why the performance changed, both in single and multi-threaded executions.

We demonstrate how TaskInsight can diagnose cases where poor scheduling caused over $60 \%$ difference on average (and up to $7 \mathrm{x}$ slowdowns) due to changes in the tasks' data reuse through the caches.
\end{abstract}

CCS Concepts • Computing methodologies $\rightarrow$ Parallel programming languages; Modeling methodologies;

Keywords Task-based Programming, Performance Modeling, Scheduling

\begin{abstract}
ACM Reference Format:
Germán Ceballos, Erik Hagersten, David Black-Schaffer. 2017. Understanding the Interplay between Task Scheduling, Memory and Performance. In Proceedings of 2017 ACM SIGPLAN International Conference on Systems, Programming, Languages, and Applications: Software for Humanity (SPLASH Companion'17). ACM, New York, NY, USA, 3 pages. https://doi.org/10.1145/3135932.3135942
\end{abstract}

Permission to make digital or hard copies of part or all of this work for personal or classroom use is granted without fee provided that copies are not made or distributed for profit or commercial advantage and that copies bear this notice and the full citation on the first page. Copyrights for thirdparty components of this work must be honored. For all other uses, contact the owner/author(s).

SPLASH Companion'17, October 22-27, 2017, Vancouver, Canada

(c) 2017 Copyright held by the owner/author(s).

ACM ISBN 978-1-4503-5514-8/17/10.

https://doi.org/10.1145/3135932.3135942

\section{Problem}

With multi-cores, new programming models have been introduced to aid the programmers dealing with the complexity of parallel systems, simplifying the coding process. Task-based programming is one popular example, where the code is split into small independent units of code (tasks) and a runtime system determines their execution order and placement. The task-based model is simpler to reason for the programmer while it is also a good approach for performance as it can adapt the scheduling to the effective resources (e.g., to the shared caches).

However, this creates a completely different dynamic of execution. There is no static scheduling determined from the code, and these executions often involve thousands of tasks that will run across different cores. This is an advantage when running on heterogeneous systems, but this irregularity along with the interplay between dynamic scheduling and caches makes hard to understand the performance of these systems, which is crucial to optimize workloads.

Traditional methods for understanding performance and bottlenecks such as cache miss ratio curves $[1,2]$ or analytical performance models [3] give results that are highly dependent on the particular task schedule and memory system (cache hierarchy and sizes). These methods work well for single-threaded programs because the memory behavior of the application does not change with the execution, but they are not a good fit for dynamic executions where the schedule changes. Thus, the analysis of two executions of the same application with different scheduling may result in different conclusions. Therefore, these techniques are not general enough to reason about task-based programs.

In this work, we present TaskInsight, a new technique to understand both the performance and memory behavior of task applications. This method can predict memory behavior in an architecturally independent way and explain changes in performance due to the interplay between scheduling and caches, enabling the developers to identify memory-related bottlenecks and to improve runtime scheduling decisions, maximizing the performance of parallel applications.

\section{Motivation and Context}

Understanding the performance of multicore systems is key for getting faster execution times and optimize workload efficiency, but it is becoming harder due to the increased complexity of both the applications and hardware architectures. Most modern systems are multicore. For example, current 


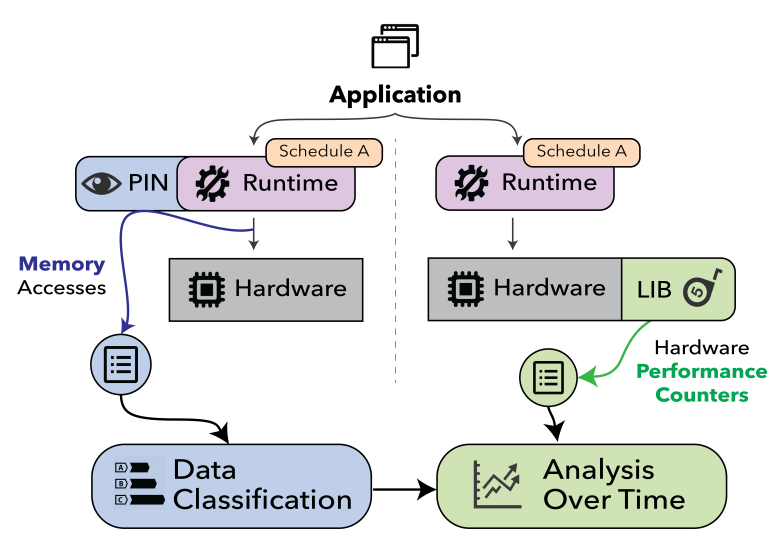

Figure 1. TaskInsight's Methodology and Implementation.

mobile phones often have 8 processors. Tasks are a relatively easy way of programming these systems, but today we do not fully understand the implications in performance of this model.

Having new techniques capable of capturing the complex nature of these systems would enable the developers to understand memory behavior of their applications and runtime systems, to reason about how it translates to performance when executing on particular systems, and to identify memory-related bottlenecks and areas for optimization.

At the same time such methods have to meet several requirements to be able to achieve this, which also represents the main challenges of this research area. First, the techniques have to be based on hardware- and executionindependent input data to avoid conflating intrinsic application behavior with system-specific effects. Second, the data collection and analysis has to be fast and low-overhead. Third, these methods should be flexible to model arbitrary architectures, memory hierarchies and schedules.

\section{Approach and Implementation}

In task-based applications, there is an intrinsic amount of data-sharing between tasks that is a function of the program structure. However, the effective amount of data sharing that will happen in the caches depends on whether the sharing tasks are scheduled close together at runtime.

We study the applications' data sharing from a locality of reference perspective, looking at two key types of data sharing: temporal and spatial. An application might reuse data brought to the cache in the past, meaning that the cache is being shared in a temporal way. On the other hand, two tasks might contend for the cache at the same moment in time, fighting to install and keep data in it, meaning that the cache is being shared in a spatial notion.

For this study, we propose a novel high-level analysis of tasks based on their sets of used data. Our tool collects information from the memory accesses and analyses data reuse over time. Data is collected from a single execution using

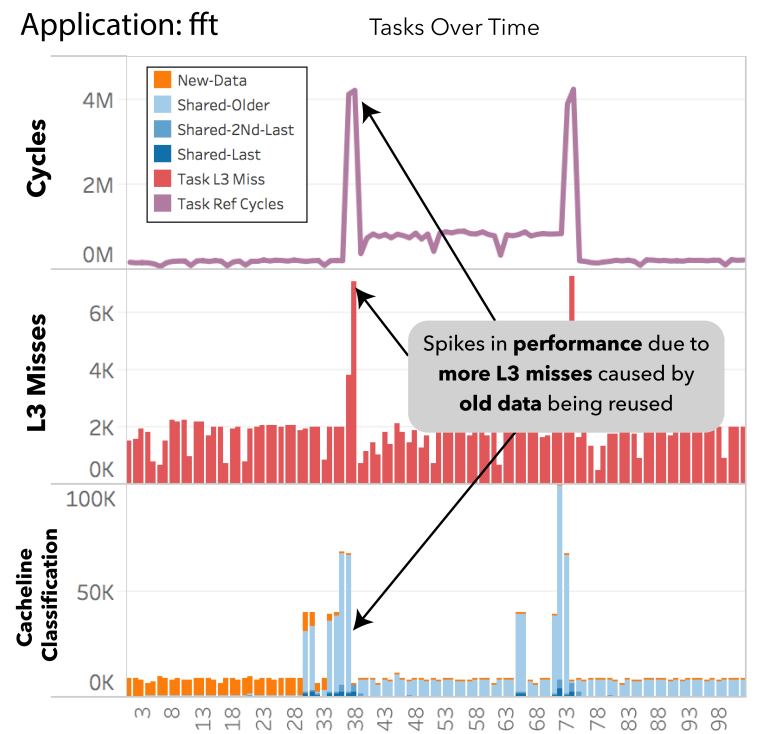

Figure 2. TaskInsight's visual feedback for developers for $\mathrm{fft}$.

binary instrumentation with Intel's Pin. The tool also collects performance results from hardware performance counters and correlates changes in performance with changes in memory behavior, while giving visual feedback to the user. A diagram of the implementation is shown in Figure 1. An example output of the tool can be seen in Figure 2, where for a particular application (fft using the OpmSs runtime), the performance of each task executed is shown over time, along with how old is the data that each task is (re)using. This allows to explain why two schedules of the sample application can result in different performance, as well as detecting the exact sources of problems: which tasks suffered and when where those executed. In fft, we see how two tasks had worse performance because they reused old data that was evicted from the cache.

\section{Results and Conclusion}

TaskInsight goes beyond previous work which typically used aggregate metrics to look at overall memory system behavior or ignored the task-level performance variation due to cache/scheduler interactions. With this deeper insight we can enable future generations of runtime and application developers, as well as schedulers to better optimize executions for complex memory systems.

TaskInsight also provides an explanation for why tasks of the same type can demonstrate significant variation in performance. As a result, programmers can now quantitatively analyze the behavior of the scheduling algorithm and the runtime can use this information to dynamically make better decisions. Our analysis exposed scheduler-induced performance differences over $10 \%$ due to $20 \%$ changes in data reuse through the private caches and up to $80 \%$ difference data reuse through the shared cache. By providing this insight we lay the groundwork for improving scheduling policies. 


\section{References}

[1] E. Berg, H. Zeffer, and E. Hagersten. 2006. A statistical multiprocessor cache model. In ISPASS. 89-99.

[2] D. Eklov and E. Hagersten. 2010. StatStack: Efficient modeling of LRU caches. In ISPASS. 55-65.

[3] Stijn Eyerman, Lieven Eeckhout, Tejas Karkhanis, and James E. Smith. 2009. A Mechanistic Performance Model for Superscalar Out-of-order Processors. ACM Trans. Comput. Syst. 27, 2, Article 3 (May 2009), 37 pages. 